\title{
Keine Zeit für die Auszeit
}

\section{Lebensarbeitszeit als Aspekt sozialer Ungleichheit}

\section{Von Philip Wotschack}

Zusammenfassung: Die Verfügbarkeit zeitlicher Ressourcen stellt einen wichtigen Aspekt der Lebensqualität und Lebenschancen dar, der in der soziologischen Ungleichheitsforschung kaum berücksichtigt wird. Es zeigt sich, dass eingeschränkte zeitliche Verfügungsmöglichkeiten in vieler Hinsicht entlang von beruflicher Stellung und Geschlecht strukturiert sind. Gering qualifizierte und weibliche Beschäftigte sind besonders stark benachteiligt. Vor diesem Hintergrund untersucht der Artikel, inwieweit lebenslauforientierte Ansätze der betrieblichen Arbeitszeitgestaltung hier zu mehr Gleichheit führen können. Im Zentrum stehen sogenannte Lebensarbeitszeit- oder Langzeitkonten. Sie bieten Beschäftigten die Möglichkeit, im Verlaufe ihres Erwerbslebens bezahlte Auszeiten für Weiterbildung, Familie oder Erholung zu realisieren. Ausgehend von der Transaktionskostentheorie werden Hypothesen zur unterschiedlichen Nutzung von Langzeitkonten nach Geschlecht und Merkmalen der beruflichen Stellung entwickelt und anhand von Regressionsanalysen empirisch überprüft. Die Ergebnisse deuten eher auf eine Kumulation als einen Abbau zeitlicher Benachteiligungen im Lebensverlauf hin.

\section{Einleitung ${ }^{1}$}

Es gehört zu den zentralen Einsichten der Zeitsoziologie, dass Zeit als soziale Kategorie zu begreifen ist, deren Strukturierung eng mit der Organisation einer Gesellschaft verknüpft ist (vgl. etwa Durkheim 1981; Sorokin / Merton 1937). Einschränkungen in der Verwendung und Strukturierung der eigenen Lebenszeit stehen in doppelter Hinsicht in einem Zusammenhang mit sozialer Ungleichheit (Wotschack 2002): Zum einen ist die Verfügung über ausreichend Zeit - für Erwerbsarbeit, Partnerschaft, Familie, soziale Kontakte oder Freizeit - heute mehr denn je ein eigenständiger Faktor der Lebensqualität (Benthaus-Apel 1995: 27; Garhammer 2007). Zum anderen bildet die Verfügung über ausreichend Zeit eine wesentliche Voraussetzung für Zeitinvestitionen - etwa in Bildung oder soziale Netzwerke - zur Erreichung beruflicher und privater Ziele. Einschränkungen bei der Verfügung über die eigene Lebenszeit sind in diesem Sinne zugleich als eine wesentliche Dimension und Determinante sozialer Ungleichheit zu begreifen.

Seit den 1980er Jahren finden sich in verschiedenen Untersuchungen Hinweise darauf, dass zeitliche Belastungen und Gestaltungsspielräume in modernen Gesellschaften ungleich verteilt sind: „Der unterschiedliche Zugriff auf Zeit unterliegt [...] den Gesetzmäßigkeiten sonstiger struktureller Ungleichheiten. Er resultiert in Ungleichheiten in der Zeitausstattung, genauer: im gänzlich unterschiedlichen Zugriff auf Umfang und Qualität der bloß scheinbar neutral und an alle gleich verteilten Ressource Zeit.“ (Müller-Wichmann 1984: 186) In verschiedenen Studien konnte eine klare Ungleichverteilung nach beruflicher Schichtung und Geschlecht nachgewiesen werden (Bauer / Groß / Schilling 1996; Garhammer 1999, 2007; BundesmannJansen / Groß / Munz 2000; Wotschack 2002; Klenner / Pfahl 2009). Beschäftigte auf den unteren Rängen der beruflichen Hierarchie - und dabei Frauen in der Regel stärker als Männer - verfügen nicht nur über geringere finanzielle Ressourcen, schlechtere Arbeits- und Lebens-

1 Für Anregungen zu Vorversionen des Textes danke ich Heike Solga (WZB), Johannes Giesecke (Otto-Friedrich-Universität Bamberg), Annette Henninger (Philipps-Universität Marburg) und Rafael Wittek (Universität Groningen). 
dingungen sowie kleinere Entscheidungsspielräume, sie sind zugleich auch häufiger in ihren zeitlichen Gestaltungsmöglichkeiten eingeschränkt.

Seit Mitte der 1990er Jahre haben unter dem Schlagwort der lebenslauforientierten Arbeitszeitgestaltung neue Ansätze Eingang in die betriebliche Arbeitszeitpolitik gefunden (vgl. Flüter-Hoffmann 2010; IW 2006). Diese verfolgen das Ziel, den Beschäftigten neue Möglichkeiten zu eröffnen, um die Erwerbsarbeitszeit an die im Lebenslauf wechselnden Betreuungs-, Pflege-, Erholungs- und Weiterbildungsbedarfe anpassen zu können. Dabei sollen auch für gering qualifizierte und weibliche Beschäftigte - also die bisher benachteiligten Gruppen bessere Möglichkeiten der Vereinbarkeit von Beruf und Familie und beruflicher Weiterbildung geschaffen werden. Besonders prominent sind sogenannte optionale Modelle, in denen die Beschäftigten individuell und flexibel auf konkrete Zeitbedarfe und Zeitinteressen im individuellen Lebenslauf reagieren können. Ein wichtiges Instrument in diesem Zusammenhang sind Ansätze, die auf eine Umverteilung der Lebensarbeitszeit zielen. So kann über sogenannte Langzeitkonten eine Umverteilung der Erwerbsarbeitszeit im Lebensverlauf ermöglicht werden: In bestimmten Lebensphasen sparen Beschäftigte Mehrarbeit oder Entgeltbestandteile auf einem langfristig angelegten Arbeitszeitkonto an. In späteren Phasen (etwa der Familienphase oder einer Phase der beruflichen Veränderung) können sie auf dieses Arbeitszeitkonto zurückgreifen und die vorgearbeitete Zeit für eigene Bedürfnisse oder Interessen nutzen, etwa für Kinderbetreuung, Pflege, Weiterbildung oder Erholung (vgl. Wotschack / Hildebrandt 2008).

Vor dem Hintergrund dieses neuen Paradigmas der Arbeitszeitgestaltung stellt sich die Frage, welche Wirkungen solche Langzeitkonten hinsichtlich der zeitlichen Gestaltungsspielräume unterschiedlicher Beschäftigtengruppen entfalten. In der aktuellen Arbeitszeitdebatte lassen sich dazu zwei gegensätzliche Auffassungen unterscheiden. Einerseits wird das große Potenzial von Langzeitkonten betont, da sie Beschäftigten bezahlte Auszeiten oder eine Reduzierung ihrer Arbeitszeit ohne Einkommensverlust ermöglichen, wovon insbesondere Beschäftigte mit geringen Einkommen und Vereinbarkeitsproblemen profitieren könnten (vgl. etwa Hildebrandt 2007). Andererseits wird die These aufgestellt, dass Langzeitkonten in erster Linie ein Instrument für Höherqualifizierte sind, da nur diese Gruppe über die nötigen zeitlichen und finanziellen Ressourcen zum Ansparen größerer Zeitguthaben verfügt (Hoff 2007).

Es stellt sich somit die Frage, welche Möglichkeiten Langzeitkonten tatsächlich für Beschäftigte eröffnen, um im Erwerbs- und Karriereverlauf Zeit für Weiterbildung und außerberufliche Anforderungen zu realisieren. Erweisen sich individuelle Lebensarbeitszeitmodelle hier in der Lage, bestehende Muster der Benachteiligung nach Geschlecht und beruflicher Stellung zu relativieren? Oder sind es wieder die unteren beruflichen Schichten und vor allem Frauen, die seltener von den Vorteilen dieses Instruments profitieren? Trifft Letzteres zu, drohen sich Ungleichheiten bei der Verfügung über Zeit im Rahmen von Lebensarbeitszeitmodellen langfristig zu verschärfen.

Die empirische Untersuchung dieser Frage steht bisher noch aus und soll im Folgenden vorgenommen werden. Eine Analyse der Nutzung von Langzeitkonten nach Unterschieden des Geschlechts und der beruflichen Stellung wurde bisher noch nicht vorgenommen und bildet den entscheidenden Forschungsbeitrag des vorliegenden Artikels. Die von der bisherigen Forschung ausgewiesenen Nutzungsbarrieren (Wotschack 2010; Seifert 2010 a; Wotschack / Hildebrandt / Scheier 2008) und Regelungsdefizite (vgl. Böker 2007; Hoff 2007) werden dabei als Ausdruck eines grundlegenden, bisher nicht gelösten, Transaktionskostenproblems verstanden, das mit der Nutzung von Langzeitkonten verbunden ist. Es entsteht im Kern dadurch, dass sich der volle Nutzen des Langzeitkontos für die Beschäftigten erst mittel- oder langfristig einstellt und damit im Prinzip unsicher ist, während die Kosten in Form von Zeit- und Geldinvestitionen bereits in der Gegenwart anfallen und in Konkurrenz zum akuten Zeit- und Geld- 
bedarf des Haushalts stehen. Mit wachsenden Risiken und Opportunitätskosten für die Beschäftigten sinkt somit der Anreiz, das Langzeitkonto zu nutzen. Trifft diese Interpretation zu, so lassen sich Unterschiede bei der Nutzung des Langzeitkontos über die damit verbundenen Risiken und Kosten unterschiedlicher Beschäftigtengruppen erklären.

Im folgenden theoretischen Teil (Abschnitt 2) wird zunächst der konzeptionelle Bezug zwischen der soziologischen Zeit- und Ungleichheitsforschung hergestellt. Aufbauend darauf werden Hypothesen zur unterschiedlichen Nutzung von Langzeitkonten nach Geschlecht und Merkmalen der beruflichen Stellung entwickelt (Abschnitt 3). Die Hypothesen werden im empirischen Teil des Artikels (Abschnitte 4 und 5) anhand von Regressionsanalysen überprüft. Für die empirischen Analysen werden Daten eines Forschungsprojekts genutzt, das von 2006 bis 2008 am Wissenschaftszentrum Berlin für Sozialforschung (WZB) durchgeführt wurde. ${ }^{2}$ Im abschließenden bilanzierenden Teil des Artikels (Abschnitt 6) werden die wichtigsten Ergebnisse zusammengefasst und Schlussfolgerungen für den zukünftigen Handlungs- und Forschungsbedarf gezogen.

\section{Lebensarbeitszeit in der Perspektive sozialer Ungleichheit}

Ungleiche Verteilungen von Ressourcen, Lebensbedingungen und Lebenschancen sind traditionell Gegenstand der soziologischen Ungleichheitsforschung. Von sozialer Ungleichheit wird gesprochen, ,,wenn Menschen [...] einen ungleichen Zugang zu sozialen Positionen haben und diese sozialen Positionen systematisch mit vorteilhaften oder nachteiligen Handlungs- und Lebensbedingungen verbunden sind“" (Solga / Powell / Berger 2009: 15; vgl. auch Solga 2005). Wesentlich ist dabei, dass diese Ungleichheiten gesellschaftlich verursacht sind, sich strukturell verfestigt haben und sich auf die Lebenschancen der Menschen auswirken, verstanden als „Chancen auf Verwirklichung von Lebenszielen, die in einer Gesellschaft im allgemeinen als erstrebenswert angesehen werden" (Geißler 1994: 4). Vor diesem Hintergrund scheint nichts dagegen zu sprechen, Zeit als Dimension und Determinante in die Ungleichheitsanalyse aufzunehmen: (1) Als eigenständiger Faktor der (objektiven und subjektiven) Lebensqualität bildet (ausreichend) Zeit eine wichtige Dimension sozialer Ungleichheit. (2) Als Voraussetzung zur Verwirklichung von Lebenschancen bildet Zeit (für Investitionen in Bildung, Karriere, Netzwerke, Partnerschaft oder Familie) eine wichtige Determinante sozialer Ungleichheit.

Während sich die Ungleichheitsforschung bisher kaum mit den alltäglichen Zeitstrukturen beschäftigt hat, haben Zeitbudgets als Indikatoren für das Wohlstandsniveau eine lange Tradition in der Zeitforschung. Schon die erste Zeitbudgetstudie in der Bundesrepublik Deutschland sah den Lebensstandard nicht nur als eine „Frage der materiellen Ausstattung einer Gesellschaft" an, sondern ,auch als eine Frage der zeitlichen Möglichkeiten für die Bevölkerung, die materiellen Möglichkeiten zu nutzen“ (v. Rosenbladt 1969: 73). Ebenso wird seit den 1990er Jahren in der bundesdeutschen Sozialstatistik und Arbeitszeitforschung die Verteilung zeitlicher Ressourcen, insbesondere der freien Zeit, als wichtiger Faktor der „menschlichen Wohlfahrt“ (Statistisches Bundesamt 1995 Bd II: 2) und des „Zeitwohlstandes“"(Bauer / Groß / Schilling 1994: 11) untersucht. Damit wird eine wesentliche Dimension sozialer Ungleichheit aufgedeckt, die im Alltag zumeist unsichtbar bleibt (vgl. Müller-Wichmann 1984; Wotschack 2002).

Für die Frage nach den zeitlichen Verfügungsmöglichkeiten spielt auch die Lage und Struktur der Zeiten im Verhältnis zu den sozialen und biologischen Zeitrhythmen eine wichtige Rolle. Ein identisches Volumen an Zeit muss nicht unbedingt das gleiche Maß an „Handlungsmöglichkeiten“" eröffnen (Müller-Wichmann 1984: 155). Freie Zeit zum falschen Zeitpunkt ist wertlos, sie kann weder für die Familie noch für kulturelle Aktivitäten oder soziale

2 Gefördert wurde das Projekt von der Hans-Böckler-Stiftung. 
Kontakte verwendet werden. Die Vereinbarkeit unterschiedlicher Lebensbereiche hängt dabei entscheidend von den betrieblichen Arbeitszeitsystemen und individuellen Arbeitszeitmustern ab. Unter Bedingungen einer zunehmenden Ausdifferenzierung und Flexibilisierung der Arbeitszeiten erhält der persönliche Einfluss auf die eigene Zeitstruktur eine herausragende Bedeutung für die Lebensqualität und die Handlungsmöglichkeiten der Menschen (Garhammer 1994: 91). Mit dem Begriff der „,relativen Zeitautonomie“ (Müller-Wichmann 1984: 177) und dem Konzept der „garantierten Optionalität“ (vgl. Hinrichs 1982) wurde auf diese wichtige Voraussetzung für die Verfügung über die eigene Lebenszeit aufmerksam gemacht. Zugleich hat die Arbeitszeitforschung wiederholt belegt, dass diese Verfügungsmöglichkeiten durch betriebliche Flexibilisierungsinteressen und geschlechtstypische Muster der Arbeitsteilung häufig eingeschränkt werden (vgl. etwa Munz 2006).

Erweitert man die soziologische Ungleichheitsforschung durch die skizzierten zeitlichen Aspekte der Lebensqualität und Lebenschancen, stellt sich die Frage, inwieweit diese entlang der Schicht- oder Geschlechtszugehörigkeit strukturiert sind.

\subsection{Eingeschränkte zeitliche Verfügungsmöglichkeiten nach beruflicher Stellung und Geschlecht}

Zeitliche Einschränkungen im Kontext von Geschlecht und Familie haben in den Sozialwissenschaften derzeit große Aufmerksamkeit (vgl. Hochschild 1990; Moen 2003; Van der Lippe / Peters 2007). Verschiedene Untersuchungen zeigen, dass sich im Zuge geschlechtstypischer Zeitrestriktionen und Zeitpräferenzen auf der Haushaltsebene ein „modernisiertes Ernährermodell“" etabliert hat (vgl. Jurczyk 2010). So leisten Frauen in allen Berufsgruppen das Gros der Haus- und Familienarbeit und weisen ein geringeres Erwerbsarbeitszeitvolumen auf. Seit den 1990er Jahren ist eine erneute Verlängerung und geschlechtsspezifische Polarisierung der Arbeitszeiten zu beobachten (Seifert 2010 b: 45): „Männer arbeiten weit überwiegend Vollzeit und durchschnittlich immer länger, Frauen zunehmend Teilzeit oder geringfügig“". Nach den Daten des Mikrozensus des Statistischen Bundesamtes von 2008 betrug die Teilzeitquote bei den abhängig beschäftigten Frauen 46\%, bei den Männern 9\%. Zugleich wenden erwerbstätige Frauen mit etwa fünf Stunden täglich im Durchschnitt etwa doppelt soviel Zeit für Reproduktionstätigkeiten auf wie Männer. Trotz der deutlich niedrigeren Erwerbszeiten verfügen sie daher in der Regel über weniger Freizeit (Statistisches Bundesamt 2003: 3; Ehling / Schwarz 1996: 10). Die bisherige Forschung belegt zudem, dass Frauen mit außerberuflichen Betreuungsaufgaben auch seltener und in geringerem Maße an beruflicher Weiterbildung teilnehmen (können) als Männer (Gillen / Elsholz / Meyer 2010).

Besonders lange Erwerbsarbeitszeiten finden sich zum einen sehr häufig bei leitenden Angestellten und Selbstständigen und dort wiederum eher bei Männern (Seifert 2003, 2007, 2009; Schupp et al. 2003). Mit einer hohen beruflichen Stellung wachsen nicht nur die Arbeitsanforderungen, auch Karrieremuster und Konkurrenz gründen sich stärker auf ein überdurchschnittliches Engagement für den Betrieb. Zugleich ist die Arbeitsorganisation hoch qualifizierter Angestellter sowohl durch ein großes Maß an Arbeitsautonomie und Teamarbeit geprägt als auch durch mehr persönliche Verantwortlichkeit und hohe, meist indirekte betriebliche Zielvorgaben (Wagner 2000). Dieses Zusammenspiel aus hoher Eigenmotivation und indirekter Kontrolle mündet in eine Arbeitszeitrealität, zu deren herausragenden Kennzeichen zeitliche Flexibilität und regelmäßige (oft unbezahlte) Mehrarbeit gehören (Seifert 2007; Kienbaum 2003, 2008). Allerdings finden sich nicht nur in Leitungspositionen hohe Erwerbsarbeitszeitvolumen. So wurde in den 1990er Jahren (abgesehen von den Selbstständigen) bei der Gruppe der Arbeiter und Arbeiterinnen am meisten Zeit durch den Erwerbsbereich gebunden (Statistisches Bundesamt 1995 Bd IV: 77ff), und auch bei niedrigen beruflichen Po- 
sitionen zeigte sich eine ausgeprägte Bereitschaft zur Mehrarbeit, die vorwiegend finanzielle Gründe hat (Groß 1998: 39ff).

Die Entgelthöhe hat entscheidenden Einfluss darauf, ob Beschäftigten zeitliche Belastungen im Alltag reduzieren können (Schupp et al. 2003). Aufgrund des geringeren Entgeltniveaus, das für eine niedrige berufliche Stellung typisch ist, führt eine Verringerung der vertraglichen Wochenarbeitszeit oder der Verzicht auf bezahlte Überstunden oft zu gravierenden finanziellen Einschnitten und gefährdet ein ausreichendes Haushaltseinkommen. Das Einkommen erweist sich hier als eine wesentliche Hürde für die Reduzierung der Erwerbsarbeitszeit (vgl. Wiesenthal 1987). In den 1990er Jahre gingen zudem mit einem hohen Haushaltseinkommen die mit Abstand niedrigsten Reproduktionszeiten einher (Schwarz 1996: 86). Durch den Kauf von Dienstleistungen und zeitsparenden Gütern lassen sich Haushaltstätigkeiten und Kinderbetreuung substituieren, und zeitliche Belastungen können reduziert werden (De Ruijter / van der Lippe 2007). So erklärt sich das Paradox eines relativ stabilen Freizeitniveaus trotz längerer Arbeitszeiten, das Mitte der 1990er Jahre für Haushalte mit einem hohen Einkommen charakteristisch war (Statistisches Bundesamt 1995 Bd II: 148ff).

Auch der Einfluss auf die alltägliche Zeitstruktur fällt beim Großteil der Beschäftigten sehr gering aus und beschränkt sich weitgehend auf Mitbestimmungsmöglichkeiten über Beginn und Ende der Arbeitszeiten, freie Tage oder verlängerte Wochenenden. Diese relative Arbeitszeitautonomie erweist sich zugleich als klar nach der beruflichen Stellung verteilt (Seifert 2007, 2009; Munz 2006). So waren Spielräume bei der Gestaltung von Lage und Dauer der eigenen Arbeitszeit Ende der 1990er Jahre in den oberen Rängen der Betriebshierarchie fast doppelt so häufig anzutreffen (25\%) wie in niedrigen Positionen (13\%) (Bundesmann-Jansen / Groß / Munz 2000: 184). Leitende Angestellte verfügten doppelt so häufig über Gleitzeitregelungen (42\%) wie einfache Angestellte (20\%) und sechsmal so häufig wie un- und angelernte Arbeiter (7\%) (vgl. Bauer et al. 1996: 150). Ein ähnliches Muster zeigt sich bei der Verbreitung von Vertrauensarbeitszeit.

Klar schichtspezifisch verteilen sich auch die Betroffenheit von Nacht- und Schichtarbeit und die damit verbundenen Belastungen durch ,unsoziale“ und schädliche Arbeitszeitlagen. Diese Ungleichheiten haben seit den 1990er Jahren zugenommen, wobei an- und ungelernte Arbeiter und Arbeiterinnen den höchsten Zuwachs verzeichnen (Bundesmann-Jansen / Groß / Munz 2000: 64ff; Seifert 2007).

Schließlich zeigen sich auch bezüglich der Verbreitung von Arbeitszeitkonten Unterschiede zwischen den Beschäftigtengruppen. Auf einem solchen Konto kann Mehrarbeit verbucht und zu einem späteren Termin in Form von arbeitsfreier Zeit kompensiert werden. Nach Zahlen des IAB verfügten 2009 etwa 32\% der bundesdeutschen Unternehmen und 50\% aller Beschäftigten über Arbeitszeitkonten (Groß / Schwarz 2007; Fischer et al. 2007: 79ff; Zapf 2012). In den Großunternehmen mit mehr als 500 Beschäftigten beträgt der Anteil der Unternehmen mit Arbeitszeitkonto mittlerweile fast $80 \%$. Innerhalb der Unternehmen sind es (neben der kleinen Gruppe der Meister und Vorarbeiter) vor allem gut qualifizierte Angestellte und Facharbeiter, die über Arbeitszeitkonten verfügen (Bauer et al. 2004). Geringqualifizierte sind klar unterrepräsentiert. Daten des Mikrozensus des Statistischen Bundesamtes (2004) deuten zudem darauf hin, dass weibliche Beschäftigte - vor allem aufgrund der Zuständigkeit für familiäre Betreuungsaufgaben - deutlich seltener in der Lage sind, Guthaben auf dem Arbeitszeitkonto aufzubauen. Sie weisen häufiger kurzfristige Zeitentnahmen auf, während Männer (insbesondere mit steigender Qualifikation) ihre Arbeitszeiten stärker an betrieblichen Arbeitserfordernissen ausrichten (vgl. Jurczyk 2010).

Insgesamt belegen die Ergebnisse eindrücklich, dass weibliche Beschäftigte und Beschäftigte mit einer niedrigen beruflichen Stellung überproportional von Einschränkungen in den 
zeitlichen Verfügungsmöglichkeiten betroffen sind. Die Folge sind Einschnitte in der Lebensqualität und den Lebenschancen der Betroffenen, die sich in Form von Zeitdruck und Zeitnot bei der Vereinbarkeit unterschiedlicher Lebensbereiche ebenso zeigen wie an fehlenden zeitlichen Ressourcen für Kinder, Weiterbildung oder berufliche Weiterentwicklung (Klenner 2004; Expertenkommission 2004). Besonders gravierende zeitliche Einschränkungen zeigen sich, wenn Benachteiligungen nach Geschlecht und beruflicher Stellung zusammenfallen (Müller-Wichmann 1984; Klenner / Pfahl 2009). Vor allem in kritischen Lebensphasen, etwa der Familienphase, Phasen beruflicher Krisen oder hoher Pflegebedarfe im Angehörigenkreis, fehlen diesen Beschäftigtengruppen oft die für die Bewältigung notwendigen zeitlichen Ressourcen (Jurczyk 2010).

\subsection{Aufhebung bestehender Benachteiligungen durch Langzeitkonten? Ein Transaktionskostenproblem}

In den letzten drei Jahrzehnten ist eine zunehmende Flexibilisierung und Differenzierung der Erwerbsarbeitszeiten zu beobachten, die sich im Spannungsfeld betrieblicher Effizienz- und Rationalisierungsstrategien einerseits und veränderter Haushaltskonstellationen und Individualisierungsprozessen andererseits vollzieht. Die Arbeitszeitgestaltung verlagerte sich stärker von der tariflichen auf die betriebliche Ebene. Neue Arbeitszeitformen wie Teilzeitarbeit, Arbeitszeitkonten, Gleitzeitarbeit oder Vertrauensarbeit verbreiteten sich. Die Arbeitszeitmuster und Arbeitszeitpräferenzen der Beschäftigten differenzierten sich weiter aus, während das Ziel kollektiver Arbeitszeitverkürzungen in den Hintergrund trat (vgl. Hinrichs 1988; Wiesenthal 1987: 109 ff). In der aktuellen Arbeitszeitdebatte werden diese Entwicklungen ambivalent bewertet: Für die Beschäftigten können sich individuelle Gestaltungsspielräume für eine bessere Vereinbarkeit der Erwerbsarbeit mit anderen Lebensbereichen eröffnen. Gleichzeitig wächst mit dem abnehmenden Regulierungsgrad der Arbeitszeit das Risiko, dass diese Spielräume von betrieblichen Flexibilitätsinteressen dominiert werden und zu neuen zeitlichen Einschränkungen für die Beschäftigten führen. So sind die Arbeitszeitmuster seit den 1990er Jahren zunehmend von Marktschwankungen und betriebswirtschaftlichen Kosten-Nutzen-Kalkülen geprägt (Seifert 2007).

Lebensarbeitszeitmodelle werden als eine mögliche Lösung für Probleme der Vereinbarkeit von beruflichen und außerberuflichen Anforderungen diskutiert. In dieser Hinsicht richtet sich das Interesse von Unternehmen, Staat und Tarifparteien besonders auf sogenannte Lebensarbeitszeit- oder Langzeitkonten. Dabei handelt es sich um Arbeitszeitkonten, mit denen über viele Jahre hinweg ein umfangreiches Zeitguthaben aufgebaut werden kann. Im Unterschied zu Arbeitszeitverkürzungen oder Teilzeitoptionen steht hier die Idee einer Umverteilung von Erwerbsarbeitszeit im Lebensverlauf im Mittelpunkt. Im Herbst 2007 boten etwa 7\% der deutschen Unternehmen Langzeitkonten an (Groß / Schwarz 2009). Bei den Großunternehmen mit mehr als 500 Mitarbeitern verfügt bereits jedes vierte Unternehmen über ein solches Langzeitkonto (Hildebrandt / Wotschack / Kirschbaum 2009). Die Grundidee von Langzeitkonten ist einfach: Beschäftigte können Überstunden oder Entgeltanteile auf einem Zeitwertkonto sparen und zu einem späteren Zeitpunkt für längere Freistellungen nutzen. Gerade in der Familienphase, wenn Kinder den Zeit- und Geldbedarf des Haushalts gleichermaßen ansteigen lassen, können Langzeitkonten von großem Vorteil sein. Sie erlauben es, phasenweise weniger zu arbeiten, ohne finanzielle Einschränkungen hinnehmen zu müssen. Ähnliches gilt für andere Ziele, wie Weiterbildung und Studium, längere Auszeiten für Erholung und Entspannung oder vorzeitigen bzw. gleitenden Ruhestand. Für weibliche Beschäftige und Beschäftigte auf den unteren Rängen der Berufshierarchie können sich auf diese Weise neue zeitliche Freiräume und Gestaltungsmöglichkeiten eröffnen und den skizzierten Benachteilungen entgegenwirken. 
Die bisherige Forschung zu Langzeitkonten hat allerdings auf eine Reihe gravierender Nutzungsbarrieren und Regelungsdefizite aufmerksam gemacht (Seifert 2010 a; Wotschack 2010; Wotschack / Hildebrandt / Scheier 2008). In der betrieblichen Praxis verfügen Beschäftigte nur eingeschränkt über die notwendige Zeitsouveränität, um den Auf- und Abbau von Arbeitszeitkonten nach ihren Interessen zu steuern (Seifert 2001; Bauer et al. 2004). Um langfristig größere Zeitguthaben aufbauen und verwenden zu können, muss in der Regel über einen längeren Zeitraum hinweg über die Regelarbeitszeit hinaus gearbeitet werden. Damit erhöhen sich nicht nur Belastungen und gesundheitliche Risiken (Nachreiner et al. 2010). Viele Beschäftigte mit außerberuflichen Versorgungsaufgaben und niedrigen Einkommen sind auch nicht in der Lage oder bereit dazu (vgl. Groß 2007; Hoff 2007). Zudem steht das Ansparen von Zeitelementen in Konkurrenz zum Arbeitseinkommen, das in der gleichen Zeit erwirtschaftet werden kann. Geldpräferenzen haben dabei eine große Dominanz (Wiesenthal 1987: 108). Negativ wirken sich schließlich auch betriebliche und überbetriebliche Regelungsdefizite aus, die den Insolvenzschutz (vgl. Fischer et al. 2007), die Übertragbarkeit von Zeitguthaben bei einem Arbeitgeberwechsel oder die Verzinsung der Zeitguthaben betreffen (vgl. Seifert 2010 a).

Diese Nutzungsbarrieren - so die zentrale These, die im Folgenden entwickelt und empirisch überprüft werden soll - lassen sich als Ausdruck eines grundlegenden Transaktionskostenproblems verstehen, das mit der Nutzung von Langzeitkonten verbunden ist und bisher nicht ausreichend im Rahmen betrieblicher und überbetrieblicher Regelungen gelöst ist: Für die Beschäftigten stellt sich der volle Nutzen des Langzeitkontos erst mittel- oder langfristig ein, während die Kosten in Form von Zeit- und Geldinvestitionen bereits in der Gegenwart anfallen. Daraus leiten sich zwei grundlegende Implikationen für das Handeln der Beschäftigten ab: (1) Je größer die Einschnitte (sprich: die Opportunitätskosten), die mit diesen Zeit- oder Geldinvestitionen verbunden sind, desto geringer ist der Anreiz, das Langzeitkonto zu nutzen. Unter Opportunitätskosten werden dabei die Kosten durch entgangene Gewinne (also der entgangene Nutzen) verstanden (vgl. Schimank 2000; Schroeter/ Rosenthal 2005). Eine zweite Implikation leitet sich aus dem Risiko des Scheiterns der geplanten Transaktion her: (2) Je größer das Risiko, dass Zeit- oder Geldinvestitionen später nicht in der gewünschten Weise verwendet werden können, umso geringer ist der Anreiz, das Langzeitkonto zu nutzen. Aus Sicht der Beschäftigten ist der langfristige Nutzen von Investitionen in das Langzeitkonto aufgrund des langen Zeithorizonts und bestehender Regelungsdefizite unsicher (insbesondere im Vergleich zu den Vorteilen kurzfristig orientierter Investitionen), während das Risiko opportunistischen Verhaltens von Seiten des Betriebes (z.B. in Form einer vorzeitigen Beendigung des Arbeitsverhältnisses oder der Verweigerung bzw. Verzögerung von Entnahmeansprüchen) hoch ist (Bolder 2002). Trifft diese Einschätzung zu, so lassen sich über das Ausmaß der jeweiligen Kosten und Risiken für unterschiedliche Beschäftigtengruppen Aussagen über die Wahrscheinlichkeit einer Nutzung des Langzeitkontos treffen.

\section{Hypothesen zur sozial ungleich verteilten Nutzung von Langzeitkonten}

Es lassen sich sechs kritische Zugangs- oder Entscheidungssituationen unterscheiden, in denen sich eine ungleiche Nutzung des Langzeitkontos vollziehen kann (siehe Tabelle 1). In den folgenden Auswertungen werden nur die Situationen zwei bis fünf untersucht. Eingeschränkte Nutzungsmöglichkeiten, die durch Unterschiede der Verbreitung und Zugänglichkeit von Langzeitkonten entstehen (Phase 1), wurden bereits an anderer Stelle untersucht (vgl. Wotschack et al. 2008). Über die tatsächliche Verwendung der entnommenen Zeit (Phase 6) liegen keine Daten vor. 
Tabelle 1: Sechs Zugangs- und Entscheidungssituationen bei der Nutzung von Langzeitkonten

\begin{tabular}{|c|c|c|c|c|c|}
\hline 1. Zugang & 2. Ansparen & 3. Volumen & 4. Präferenz & 5. Entnahme & 6. Zweck \\
\hline $\begin{array}{l}\text { Gibt es ein } \\
\text { Langzeit- } \\
\text { konto im Be- } \\
\text { trieb? Darf es } \\
\text { genutzt wer- } \\
\text { den? }\end{array}$ & $\begin{array}{l}\text { Wurde min- } \\
\text { destens ein- } \\
\text { mal Gutha- } \\
\text { ben einge- } \\
\text { stellt? }\end{array}$ & $\begin{array}{l}\text { In welchem } \\
\text { Umfang } \\
\text { wird Zeit } \\
\text { angespart? }\end{array}$ & $\begin{array}{l}\text { Wofür soll } \\
\text { die gesparte } \\
\text { Zeit ver- } \\
\text { wendet wer- } \\
\text { den? }\end{array}$ & $\begin{array}{l}\text { Kann Zeit } \\
\text { nach } \\
\text { Wunsch ent- } \\
\text { nommen } \\
\text { werden? }\end{array}$ & $\begin{array}{l}\text { Wofür wird } \\
\text { die Zeit ver- } \\
\text { wendet? }\end{array}$ \\
\hline
\end{tabular}

\section{Ansparen (Phase 2) und Volumen (Phase 3) auf dem Langzeitkonto}

Mit dem Blick auf dem Ansparprozess stehen die damit verbundenen Opportunitätskosten im Mittelpunkt: Jede Minute und jeder Entgeltbestandteil, die auf dem Langzeitkonto für einen späteren Verwendungszweck gespart werden, stehen den Beschäftigten im Alltag nicht mehr zur Verfügung, können also nicht direkt in Haushaltseinkommen oder Zeit (etwa für Familie, Betreuungsaufgaben, Erholung oder Freizeit) umgewandelt werden. Diese Opportunitätskosten wachsen in dem Maße, in dem Beschäftigte auf die unmittelbare Nutzung von Zeit- und Entgeltbestandteile angewiesen sind. Das gilt, wie oben gezeigt, zum einen für Beschäftigte mit einer niedrigeren beruflichen Stellung. Aufgrund der geringeren Vergütung sind sie auf die unmittelbare und vollständige finanzielle Entgeltung ihrer Arbeitszeit angewiesen, um ein ausreichendes Haushaltseinkommen zu erzielen. Dementsprechend ist zu erwarten, dass Beschäftigte mit einer niedrigeren beruflichen Stellung entsprechend seltener und weniger Guthaben auf dem Langzeitkonto ansparen. Es hat sich zudem gezeigt, dass ein großes Volumen an unbezahlter Mehrarbeit ein typisches Merkmal der Arbeitssituation von Beschäftigten mit hoher beruflicher Stellung ist. Daher ist zu erwarten, dass das Langzeitkonto für diese Gruppe eher die Möglichkeit eröffnet, (zumindest) einen Teil dieser ohnehin anfallenden Mehrarbeit auf dem Langzeitkonto zu sparen, ohne dass damit zusätzliche finanzielle oder zeitliche Einschränkungen im Alltag verbunden sind. Aus diesen Überlegungen lässt sich die folgende Hypothese ableiten:

(H1) Beschäftigte mit einer niedrigeren Stellung im Beruf sparen seltener und in geringerem Maße Guthaben auf dem Langzeitkonto an.

Auch für Beschäftigte mit höheren außerberuflichen Verpflichtungen führt das Ansparen von Zeitguthaben zu stärkeren Einschnitten im Alltag, sprich: ist mit höheren Opportunitätskosten verbunden. Das trifft, wie oben gezeigt, häufiger auf weibliche Beschäftigte zu, da sie durch die geschlechtstypische Arbeitsteilung vorrangig für Haus- und Familienarbeit zuständig sind. Folglich erwarten wir, dass weibliche Beschäftigte seltener und in geringerem Maße die dafür nötige Zeit auf dem Langzeitkonto ansparen (können), da sie diese Zeit akut im Alltag für die Vereinbarkeit von Erwerbs-, Haus- und Familienarbeit benötigen. Das ist besonders für Vollzeit beschäftigte Frauen zu erwarten sowie für weibliche Beschäftigte in der mittleren Lebensphase, bei denen häufiger Aufgaben der Kinderbetreuung gegeben sind. Daraus lässt sich die folgende Hypothese ableiten:

(H2) Weibliche Beschäftigte sparen seltener und in geringerem Maße Guthaben auf dem Langzeitkonto an, besonders wenn sie Vollzeit erwerbstätig sind oder sich in der mittleren Lebensphase befinden. 
Eine wichtige Voraussetzung für die Vereinbarkeit der Erwerbsarbeit mit außerberuflichen Anforderungen und Interessen stellt der Einfluss auf die eigene Arbeitszeitstruktur dar. Beschäftigte, die mit externer Arbeitszeiterfassung und direkter Arbeitszeitkontrolle arbeiten, verfügen formal über weniger Autonomie bei der individuellen Arbeitszeitgestaltung. Sie sollten daher ein höheres Interesse daran haben, die mit dem Langzeitkonto verbundene Möglichkeit des Zeitausgleichs in Form bezahlter Auszeiten zu nutzen. Im Vergleich zu den (in der Regel höher qualifizierten) Beschäftigten mit Vertrauensarbeitszeit verfügen sie allerdings über geringere finanzielle Ressourcen für den Aufbau von Guthaben auf dem Langzeitkonto. Daraus lässt sich die folgende Hypothese ableiten:

(H3) Beschäftigte mit (externer) Arbeitszeiterfassung sparen häufiger Guthaben auf dem Langzeitkonto an als Beschäftigte in Vertrauensarbeitszeit, verfügen aber über geringere Zeitguthaben.

Schließlich lassen sich Vorhersagen über die Nutzung des Langzeitkontos aus dem (antizipierten) Risiko des Scheiterns einer späteren Zeitentnahme herleiten. Ein solches Risiko stellen, wie oben gezeigt, die vorzeitige Beendigung des Arbeitsverhältnisses dar sowie die Verweigerung der gewünschten Zeitentnahme oder des gewünschten Zeitpunktes durch den Vorgesetzten. Mit zunehmender Unsicherheit der eigenen Situation im Betrieb wächst das Risiko, dass kein langfristiges Guthaben aufgebaut und zu einem späteren Zeitpunkt genutzt werden kann. Beschäftigte, die für sich selbst keine langfristige Perspektive im Betrieb sehen, sollten daher das Langzeitkonto seltener nutzen.

(H4) Je unsicherer die eigene Zukunft im Betrieb wahrgenommen wird, umso seltener wird auf dem Langzeitkonto angespart.

Treffen die formulierten Hypothesen zu, würden Beschäftigte mit einer niedrigen Stellung im Beruf, weibliche Beschäftigte (in bestimmten Arbeits- und Lebenskontexten) und Beschäftigte mit einer unsicheren Zukunftsperspektive im Unternehmen in geringerem Maße Guthaben auf Langzeitkonten ansparen und nutzen können.

\section{Verwendungspräferenz (Phase 4) und Entnahme (Phase 5) von Zeitguthaben}

Für Beschäftigte, die bereits über Guthaben auf dem Langzeitkonto verfügen, stellt sich die Frage, wie sie es einsetzen und verwenden möchten. Eine eher zeitnahe Nutzung des Zeitguthabens (etwa für Weiterbildung oder Familie) steht dabei in Konkurrenz zum langfristigen Ansparen der Zeit (etwa für den Vorruhestand). Sind Beschäftigte aufgrund finanzieller Restriktionen (bei niedriger Stellung im Beruf) oder hoher außerberuflicher Verpflichtungen (bei weiblichen Beschäftigten) in den zeitlichen Verfügungsmöglichkeiten eingeschränkt, eröffnet ein vorhandenes Guthaben auf dem Langzeitkonto die Möglichkeit einer bezahlten Auszeit für Aktivitäten, die sonst im Alltag zu kurz kommen. Bei diesen Beschäftigtengruppen sind die Opportunitätskosten eines langfristigen Ansparens (in Form eines Verzichts auf eine zeitnahe Entnahme) somit höher als bei Beschäftigten, die im Alltag über größere zeitliche und finanzielle Spielräume verfügen. Wenn man zusätzlich davon ausgeht, dass mit einer niedrigen Stellung im Beruf auch das Arbeitsplatzrisiko wächst, erhöht langfristiges Ansparen zugleich das Risiko, dass die gewünschte Zeitentnahme durch eine vorzeitige Beendigung des Arbeitsverhältnisses verhindert werden kann. Entsprechend ist zu erwarten, dass Beschäftigte mit 
niedriger Stellung im Beruf, ein vorhandenes Zeitguthaben eher zeitnah nutzen wollen. Daraus lässt sich die folgende Hypothese ableiten:

(H5) Verfügen Beschäftigte mit einer niedrigeren Stellung im Beruf über ein Langzeitkonto, entnehmen sie häufiger Zeit für Familie oder Weiterbildung.

Auch für weibliche Beschäftigte ist das langfristige Ansparen von Zeit im Vergleich zu männlichen Beschäftigten mit höheren Opportunitätskosten verbunden. Daher sollten sie ein vorhandenes Zeitguthaben eher zeitnah für Aktivitäten wie Familienzeit oder Weiterbildung einsetzen wollen. Das gilt besonders für Vollzeit beschäftigte Frauen und Frauen in der mittleren Lebensphase, bei denen die Wahrscheinlichkeit einer hohen Belastung durch Beruf und Haushalt allgemein höher ist. Daraus lässt sich die folgende Hypothese ableiten:

(H6) Verfügen weibliche Beschäftigte über ein Langzeitkonto, entnehmen sie häufiger Zeit für Familie oder Weiterbildung.

\section{Methoden: Forschungsdesign, Sample, Variablen}

Die verwendeten Daten stammen aus dem Projekt „Langzeitkonten und biographische Lebensführung“, das von 2006 bis 2008 am Wissenschaftszentrum Berlin für Sozialforschung durchgeführt wurde. Die wichtigste Datenbasis stellen dabei Daten aus einer Beschäftigtenbefragung in einem großen Verkehrsdienstleistungsbetriebs (mit mehr als 10.000 Beschäftigten) sowie Personal- und Zeiterfassungsdaten eines mittelgroßen IT-Betriebs (mit weniger als 1.000 Beschäftigten) dar. Die Betriebe sind keinesfalls repräsentativ. Beide haben Vorreitercharakter und bieten ihren fest angestellten Mitarbeitern im Rahmen betrieblicher Vereinbarungen bereits seit mehreren Jahren die Möglichkeit, ein Langzeitkonto zu führen und Zeit für individuelle Zwecke zu entnehmen. Die betrieblichen Rahmenregelungen (etwa beim Insolvenzschutz oder der Verzinsung) und formalen Nutzungsmöglichkeiten sind in beiden Fällen als relativ günstig einzustufen und werden auch von den betrieblichen Arbeitnehmervertretungen mitgetragen. Gemäß der zentralen Untersuchungsfrage werden Unterschiede bei der Nutzung des Langzeitkontos nach Merkmalen der beruflichen Stellung und des Geschlechts analysiert. Andere Einflussfaktoren, wie branchen- und geschlechtstypische Arbeitszeitkulturen, sollen damit keineswegs geleugnet werden, können aber nicht in die Untersuchung einbezogen werden (vgl. dazu Wotschack / Hildebrandt 2008). Repräsentative Individualdaten zur Nutzung von Langzeitkonten, die es möglich machen würden, auch den Einfluss branchenund betriebsspezifischer Merkmale einzubeziehen, liegen bisher leider nicht vor. Auch zu der Haushaltssituation der Beschäftigten enthalten die verwendeten Betriebsdaten leider keine Informationen.

\section{Der Verkehrsdienstleistungsbetrieb - abhängige und unabhängige Variablen}

In dem Verkehrsdienstleistungsbetrieb wurden 2007 im Rahmen einer jährlich stattfindenden repräsentativen Mitarbeiterbefragung auch Fragen zur Nutzung des Langzeitkontos gestellt. Diese bilden die abhängigen Variablen und beziehen sich auf das Ansparen mit dem Langzeitkonto: „Nutzen Sie das Langzeitkonto?“(Antwortkategorien waren ,ja“ oder ,nein“) sowie auf die gewünschte Verwendung des Zeitguthabens: „Welche Verwendung der angesparten Zeit bevorzugen Sie?“ (Antwortkategorien waren: „Weiterbildung“, „Sabbatical“, „Familie“, „vorübergehende Teilzeit“ und „Verkürzung der Lebensarbeitszeit“). Als unabhängige Variablen wurden entsprechend den formulierten Hypothesen folgende Merkmale der Beschäftigten 
in die Auswertungen einbezogen: Geschlecht, berufliche Stellung (Funktion im Betrieb) sowie die antizipierte Unsicherheit der eigenen Zukunft im Unternehmen. Für Letztere wurde ein Index aus vier Items gebildet. Dieser basiert auf der Selbsteinschätzung der Beschäftigten (1 „trifft überhaupt nicht zu“ bis 6 ,trifft völlig zu“) in den Bereichen (1) Arbeitsplatzsicherheit („Habe Gefühl von Arbeitsplatzsicherheit“), (2) Entwicklungsmöglichkeiten im Unternehmen („Habe gute berufliche Entwicklungsmöglichkeiten“), (3) Mitarbeiterengagement der Vorgesetzten (,Vorgesetzte kümmern sich gut um Mitarbeiter“) und (4) Vertrauen in die Unternehmensleitung („Habe Vertrauen in die Unternehmenspolitik“). Geringe Zustimmung (Werte 1 und 2) in den einzelnen Bereichen wurde gezählt und aufaddiert (Cronbach's Alpha ist.77). Aus Gründen der Anonymisierung wurden in der Mitarbeiterbefragung keine Informationen über das Alter und die Haushaltssituation der Beschäftigten erfasst.

An der Befragung haben 5.476 Beschäftigte teilgenommen, von denen 5.300 in die Auswertung einbezogen wurden. Es bestand leider keine Möglichkeit Ausfall- und Selektivitätsanalysen durchzuführen. Alle Befragten haben ein unbefristetes Beschäftigungsverhältnis. Fast zwei Drittel von ihnen (61\%) sind als einfache Angestellte im so genannten operativen Bereich tätig. Dabei handelt es sich um Tätigkeiten mit geringen Qualifikationsanforderungen im Bereich Be- und Entladen, Transport und Sicherheit, die im Schichtbetrieb ausgeführt werden. Mehr als ein Drittel der Befragten (39\%) arbeitet als qualifizierte Angestellte im so genannten administrativen Bereich und übt dort Tätigkeiten in der Verwaltung, Koordinierung oder im Marketing aus. Für diese Beschäftigten gilt ein Gleitzeitmodell. Vier Fünftel der Befragten sind Männer, ein Fünftel Frauen. Der Frauenanteil beträgt im operativen Bereich etwa $10 \%$, im administrativen Bereich etwa ein Drittel.

\section{Der IT-Betrieb - abhängige und unabhängige Variablen}

Die Auswertungen für das IT-Unternehmen beruhen nicht auf Befragungsdaten, sondern auf Personal- und Zeiterfassungsdaten für den Zeitraum von (November) 2001 bis (November) 2007. Die Daten des Unternehmens geben für diesen Zeitraum genaue Informationen über die jährlichen Kontenbewegung. Für die Analyse sind die folgenden drei Dimensionen der Kontennutzung relevant (abhängige Variablen):

1. Nutzung des Langzeitkontos: Wurde zwischen 2001 und 2007 mindestens einmal Zeit oder Geld in das Langzeitkonto eingespeist?

2. Kontenvolumen nach sieben Jahren: Wie hoch ist das Zeitguthaben auf dem Langzeitkonto am Ende des Beobachtungszeitraums (Ende 2007)?

3. Entnahme von Zeitguthaben: Wurde zwischen 2001 und 2007 mindestens einmal Zeit entnommen?

Für die Beschäftigten des untersuchten IT-Unternehmens sind ein hohes Qualifikationsniveau ( $85 \%$ haben einen Hochschulabschluss), eine lange Beschäftigungsdauer und eine unbefristete Anstellung charakteristisch. Der Altersdurchschnitt ist mit 44 Jahren relativ hoch. Der Frauenanteil liegt bei $30 \%$, der Anteil von Teilzeitbeschäftigten bei $16 \%$. Im Unternehmen wird entweder mit Vertrauensarbeitszeit (keine Zeiterfassung, dafür hohe Bedeutung von variablen Gehaltsbestandteilen in Form von Boni oder Prämien) gearbeitet oder in einem Gleitzeitmodell mit Zeiterfassung. In das Langzeitkonto können sowohl variable Gehaltsbestandteile (Prämien, Boni) eingestellt werden als auch Zeitguthaben (Mehrarbeit) vom Gleitzeitkonto. Als unabhängige Variablen werden entsprechend den formulierten Hypothesen folgende Merkmale der Beschäftigten einbezogen: Geschlecht, Vergütung (Stundensatz), Beschäftigungsverhältnis (Vollzeit oder Teilzeit), Arbeitszeitmodell (Gleitzeit oder Vertrauensarbeitszeit) und Lebensphase (vier Altersgruppen). Da keine Informationen zur Haushaltkonstellation vorliegen, wird als Behelf die Variable Alter bzw. Lebensphase genutzt und angenommen, dass die Wahr- 
scheinlichkeit von (kleineren) Kindern im Haushalt bei jüngeren Beschäftigten (jünger als 46 Jahre) allgemein höher ist.

\section{Analyse: Ansparen und Verwendung von Zeit auf dem Langzeitkonto}

Die Hypothesen werden im Folgenden anhand der Beschäftigtendaten aus den zwei Betrieben überprüft. Es werden die wichtigsten Ergebnisse aus den deskriptiven (Abschnitt 5.1) und multivariaten (Abschnitt 5.2) Auswertungen der Beschäftigtendaten vorgestellt. Die statistischen Analysen beruhen auf logistischen und linearen Regressionsanalysen.

\subsection{Deskriptive Analyse: Häufigkeitsverteilungen und Mittelwerte}

Werden die Anteile der Nutzer / innen des Langzeitkontos in den beiden Befragungsbetrieben gegenübergestellt, zeigen sich deutliche Unterschiede nach der Stellung im Beruf. Der Verbreitungsgrad des Langzeitkontos ist bei der vorwiegend aus hoch qualifizierten Angestellten bestehenden Belegschaft des IT-Unternehmens (mit 80\%) wesentlich höher als bei der aus einfachen und qualifizierten Angestellten bestehenden Belegschaft des Verkehrsdienstleistungsunternehmens (mit 27\%).

Bei den einfachen Angestellten im operativen Bereich des Verkehrsdienstleistungsbetriebs wird das Konto am seltensten genutzt (siehe Tabelle 2). Bezüglich des Aufbaus von Guthaben auf dem Langzeitkonto zeigt sich zudem, dass höhere Vergütungsgruppen und ältere Beschäftigte nicht nur häufiger über ein Langzeitkonto verfügen, sondern auch über die höchsten Guthaben (siehe Tabelle 3). Bei der Entnahme von Zeit nutzen hingegen weibliche Beschäftigte, Beschäftigte in der mittleren Lebensphase und mittlere Vergütungsgruppen das Konto am häufigsten.

Tabelle 2: Nutzung des Langzeitkontos und Verwendungspräferenzen im Verkehrsdienstleistungsbetrieb (in \%)

\begin{tabular}{|c|c|c|c|c|}
\hline & \multirow[t]{2}{*}{ Nutzung } & \multicolumn{3}{|c|}{ Motiv } \\
\hline & & $\begin{array}{l}\text { Weiterbil- } \\
\text { dung* }\end{array}$ & $\begin{array}{l}\text { Familien- } \\
\text { zeit* }\end{array}$ & $\begin{array}{l}\text { Vorruhe- } \\
\text { stand* }\end{array}$ \\
\hline Alle $(n=5300)$ & 27 & 18 & 33 & 55 \\
\hline $\begin{array}{l}\text { Administrativer Bereich } \\
\text { Frauen } \\
\text { Männer }\end{array}$ & $\begin{array}{l}38 \\
40\end{array}$ & $\begin{array}{l}25 \\
18\end{array}$ & $\begin{array}{l}32 \\
27\end{array}$ & $\begin{array}{l}56 \\
67\end{array}$ \\
\hline $\begin{array}{l}\text { Operativer Bereich } \\
\text { Frauen } \\
\text { Männer }\end{array}$ & $\begin{array}{l}18 \\
19\end{array}$ & $\begin{array}{l}24 \\
14\end{array}$ & $\begin{array}{l}42 \\
37\end{array}$ & $\begin{array}{l}44 \\
45\end{array}$ \\
\hline $\begin{array}{l}\text { Unsichere Zukunft (Selbsteinschät- } \\
\text { zung) } \\
\text { Kaum berufliche Entwicklungsmöglich- } \\
\text { keiten }\end{array}$ & 26 & 16 & 34 & 54 \\
\hline $\begin{array}{l}\text { Kaum Gefühl von Arbeitsplatzsicherheit } \\
\text { Kaum Gefühl, dass Vorgesetzte sich } \\
\text { kümmern }\end{array}$ & $\begin{array}{l}24 \\
25\end{array}$ & $\begin{array}{l}15 \\
14\end{array}$ & $\begin{array}{l}36 \\
34\end{array}$ & $\begin{array}{l}52 \\
54\end{array}$ \\
\hline $\begin{array}{l}\text { Kaum Vertrauen in die Unternehmens- } \\
\text { leitung }\end{array}$ & 26 & 16 & 35 & 55 \\
\hline
\end{tabular}

* Die Werte beziehen sich auf die Beschäftigten, die das Langzeitkonto nutzen. 
Tabelle 3: Nutzung des Langzeitkontos im IT-Betrieb

\begin{tabular}{|c|c|c|c|}
\hline & $\begin{array}{l}\text { Nutzung } \\
\text { (in \%) }\end{array}$ & $\begin{array}{l}\text { Kontenvolumen } \\
\text { (Stundenmittelwerte)* }\end{array}$ & $\begin{array}{l}\text { Mindestens eine } \\
\text { Entnahme (in \%)* }\end{array}$ \\
\hline Alle $(n=745)$ & 80 & 168 & 32 \\
\hline $\begin{array}{l}\text { Geschlecht } \\
\text { Frauen } \\
\text { Männer }\end{array}$ & $\begin{array}{l}80 \\
81\end{array}$ & $\begin{array}{l}152 \\
175\end{array}$ & $\begin{array}{l}38 \\
29\end{array}$ \\
\hline $\begin{array}{l}\text { Beschäftigungsverhäl } \\
\text { Vollzeit } \\
\text { Teilzeit } \\
\end{array}$ & $\begin{array}{l}79 \\
88 \\
\end{array}$ & $\begin{array}{l}173 \\
143 \\
\end{array}$ & $\begin{array}{l}31 \\
35 \\
\end{array}$ \\
\hline $\begin{array}{l}\text { Alter } \\
\text { 19-35 Jahre } \\
\text { 36-45 Jahre } \\
\text { 46-55 Jahre } \\
\text { 56-64 Jahre } \\
\end{array}$ & $\begin{array}{l}38 \\
79 \\
91 \\
91 \\
\end{array}$ & $\begin{array}{l}97 \\
160 \\
169 \\
219 \\
\end{array}$ & $\begin{array}{l}22 \\
37 \\
31 \\
24 \\
\end{array}$ \\
\hline $\begin{array}{l}\text { Stundensatz } \\
\text { Unter 20,10 Euro } \\
\text { 20,10-30,00 Euro } \\
\text { 30,10-40,00 Euro } \\
\text { 40,10-60,00 Euro }\end{array}$ & $\begin{array}{l}63 \\
80 \\
85 \\
83\end{array}$ & $\begin{array}{l}113 \\
131 \\
183 \\
275\end{array}$ & $\begin{array}{l}25 \\
40 \\
29 \\
25\end{array}$ \\
\hline $\begin{array}{l}\text { Arbeitszeitmodell } \\
\text { Arbeitszeiterfassung } \\
\text { Vertrauensarbeitszeit }\end{array}$ & $\begin{array}{l}88 \\
69 \\
\end{array}$ & $\begin{array}{l}131 \\
245\end{array}$ & $\begin{array}{l}29 \\
39\end{array}$ \\
\hline
\end{tabular}

* Die Werte beziehen sich auf die Beschäftigten, die das Langzeitkonto nutzen.

Unterschiede zwischen Männern und Frauen sind sowohl bei der Verbreitung des Langzeitkontos als auch bei den Verwendungsinteressen nur geringfügig ausgeprägt. Von den Beschäftigten, die über ein Langzeitkonto verfügen, spart die Mehrheit (55\%) für eine Verkürzung der Lebensarbeitszeit (Vorruhestand) an. Im administrativen Bereich ist dieser Wunsch bei den Männern besonders stark verbreitet. Immerhin ein Drittel (33\%) der Befragten möchte das Zeitguthaben für die Familie nutzen, Frauen häufiger als Männer. Ein knappes Fünftel (18\%) der Befragten gibt an, die Zeit für Weiterbildung nutzen zu wollen. Auch hier sind es häufiger Frauen, die diesen Verwendungswunsch äußern.

\subsection{Regressionsanalyse: Ansparen mit dem Langzeitkonto}

Die Regressionsanalysen belegen einen deutlichen, signifikanten Einfluss der beruflichen Stellung auf die Verbreitung des Langzeitkontos und die Höhe der Guthaben: Die qualifizierten Angestellten im administrativen Bereich des Verkehrsdienstleistungsbetriebs verfügen wesentlich häufiger über ein Langzeitkonto als die einfachen Angestellten des operativen Bereichs (siehe Anhang Tabelle 4, Modell I). Dieser Zusammenhang existiert unabhängig vom Geschlecht der Befragten. Im IT-Betrieb (siehe Anhang Tabelle 5, Modell I) zeigen sich hinsichtlich der Verbreitung des Langzeitkontos signifikante Unterschiede nach der Vergütungshöhe. Beschäftigte mit einem niedrigeren Stundensatz (30 Euro und weniger) verfügen (signifikant) seltener über ein Langzeitkonto als Beschäftigte mit höherer Vergütung. Sie haben zudem (signifikant) niedrigere Guthaben. ${ }^{3}$ Insgesamt bestätigt sich damit die Hypothese $\mathrm{HI}$ : Die mit einer niedrigen beruflichen Stellung verbundenen Restriktionen wirken sich nachteilig auf den Aufbau von Guthaben mit dem Langzeitkonto aus.

3 Aus Platzgründen können die entsprechenden Regressionstabellen zum Kontenvolumen hier nicht dokumentiert werden. Sie können beim Autor angefordert werden. 
Signifikante Unterschiede zwischen Männern und Frauen zeigen sich hingegen weder hinsichtlich der Verbreitung des Langzeitkontos noch hinsichtlich der Höhe des Guthabens - auch nicht, wenn zusätzlich das jeweilige Beschäftigungsverhältnis (Vollzeit- oder Teilzeitbeschäftigung) berücksichtigt wird. Allerdings finden sich für die Kombinationen der Merkmale Geschlecht und Alter bzw. Geschlecht und Vergütungshöhe zumindest Hinweise darauf, dass Frauen in bestimmten Lebensphasen und bei finanziellen Restriktionen benachteiligt sind: Jüngere Frauen (unter 46 Jahren) verfügen seltener über ein Langzeitkonto (siehe Anhang Tabelle 5, Modell IV). Für Frauen mit geringerer Vergütung (weniger als 30 Euro pro Stunde) sind besonders niedrige Guthaben auf dem Lebensarbeitskonto charakteristisch. Diese Effekte sind nur auf einem 10\%-Niveau signifikant. Die Hypothese H2 bestätigt sich damit nur teilweise.

Bei Beschäftigten mit (externer) Zeiterfassung (Gleitzeit) ist die Chance, dass sie Zeit auf dem Langzeitkonto ansparen, deutlich höher als bei Beschäftigten mit Vertrauensarbeitszeit (siehe Anhang Tabelle 5; Modell I). Andererseits weisen sie signifikant niedrigere Zeitguthaben auf als Beschäftigte in Vertrauensarbeitszeit. Damit bestätigt sich die Hypothese H3: Bei externer Arbeitszeitkontrolle (Zeiterfassung) stellt das Langzeitkonto ein wichtiges Instrument für den Ausgleich von Mehrarbeitszeit dar und wird daher häufiger genutzt. Zugleich sind aber die Möglichkeiten, über Mehrarbeit größere Guthaben anzusparen, deutlich geringer als bei den (in der Regel höher qualifizierten) Beschäftigten in Vertrauensarbeitszeit. Auch Beschäftigte, die ihre Zukunft im Unternehmen eher als unsicher wahrnehmen, nutzen seltener das Langzeitkonto (Tabelle 4, Modell II). Damit bestätigt sich Hypothese H4.

\subsection{Regressionsanalyse: Entnahme und gewünschte Verwendung der Zeit}

Es findet sich kein signifikanter Zusammenhang zwischen einer niedrigeren beruflichen Stellung oder Vergütung und der Wahrscheinlichkeit einer Entnahme von Zeit vom Langzeitkonto. ${ }^{4}$ Der erste Teil der Hypothese $H 5$ kann damit nicht bestätigt werden. Allerdings zeigt sich bezüglich der gewünschten Verwendung des Guthabens auf dem Langzeitkonto, dass Beschäftigte im administrativen Bereich (und dabei vor allem Männer) häufiger die Verkürzung der Lebensarbeitszeit (Vorruhestand) angeben und seltener Familienzeit. Beschäftigte im operativen Bereich, die über ein Langzeitkonto verfügen, haben hingegen häufiger ein Interesse daran, das Guthaben für Familienzeit zu verwenden. Damit bestätigt sich der zweite Teil der Hypothese H5 für den Verwendungswunsch Familienzeit (allerdings nicht für Weiterbildung). Bei den Beschäftigten des administrativen Bereichs und dabei besonders bei den Männern wird das Langzeitkonto dagegen eher als Option genutzt, um langfristig für den vorzeitigen Ausstieg aus dem Erwerbsleben zu sparen.

Weibliche Beschäftigte haben im Beobachtungszeitraum häufiger Zeit als Männer entnommen (der Effekt ist auf 10\%-Niveau signifikant). Die vertiefende Analyse unterschiedlicher Kontextfaktoren (Interaktionen) zeigt, dass es sich dabei vor allem um Vollzeit beschäftigte Frauen handelt. Damit bestätigt sich der erste Teil der Hypothese H6: Frauen, die über ein Langzeitkonto verfügen, nutzen das Guthaben häufiger zur zeitlichen Entlastung ihres Alltags. Einen Beleg für den erwarteten Einfluss der Lebensphase findet sich allerdings nicht.

Die Analysen zeigen zudem, dass weibliche Beschäftigte das Langzeitkonto häufiger für Weiterbildung nutzen möchten als ihre männlichen Kollegen. Damit bestätigt sich die Hypothese $H 6$ zumindest bezüglich des Verwendungswunsches für Weiterbildung; für den Wunsch nach Familienzeit finden sich keine signifikanten Unterschiede zwischen Männern und Frauen.

4 Aus Platzgründen können die entsprechenden Regressionstabellen zum Kontenvolumen hier nicht dokumentiert werden. Sie können beim Autor angefordert werden. 


\section{Diskussion und Schlussfolgerungen}

Befunde der bisherigen Zeit- und Ungleichheitsforschung belegen klar, dass Unterschiede in der Verfügung über Zeit in vieler Hinsicht parallel zu Ungleichheiten nach beruflicher Schichtung und Geschlecht verlaufen. Weibliche Beschäftigte und Beschäftigte mit einer niedrigen beruflichen Stellung sind besonders oft mit Einschränkungen konfrontiert, wenn sie ihre Erwerbstätigkeit mit ausreichend Zeit für Kinderbetreuung, Pflege, Weiterbildung, Familie oder Freizeitaktivitäten verbinden wollen. Als große Hindernisse erweisen sich dabei zum einen das für eine niedrigere berufliche Stellung typische geringere Entgeltniveau und zum anderen rigide Arbeitszeitstrukturen mit geringen individuellen Einflussmöglichkeiten.

Die hier vorgestellten Analysen von Beschäftigtendaten aus zwei Dienstleistungsbetrieben lassen es fraglich erscheinen, dass Langzeitkonten in der Lage sind, diese Ungleichheiten zu relativieren. Die Befunde deuten für Frauen und Beschäftigten mit niedriger beruflicher Stellung eher auf eine doppelte Benachteiligung hinsichtlich der zeitlichen Verfügungsmöglichkeiten hin: Sie sind im gegenwärtigen Zugriff auf Zeit ebenso eingeschränkt wie in den Möglichkeiten, sich langfristig größere Zeitguthaben aufzubauen. Diese Probleme können, wie gezeigt, als Ausdruck eines grundlegenden, bisher nicht gelösten, Transaktionskostenproblems interpretiert werden: Je weniger Zeit- und Geldinvestitionen in der Gegenwart geleistet werden können (etwa durch zeitliche und finanzielle Restriktionen) und je unsicherer deren zukünftiger Nutzen ist (etwa durch Unsicherheit der eigenen Situation im Betrieb), umso geringer ist der Anreiz, das Langzeitkonto zu nutzen. Bei Beschäftigten mit niedriger beruflicher Stellung und weiblichen Beschäftigten in bestimmten Lebenskontexten sind diese Transaktions- und Opportunitätskosten im Vergleich zu anderen Beschäftigtengruppen deutlich höher. In der Folge nutzen sie das Instrument in geringerem Maße. Hinsichtlich der Wirkung des Langzeitkontos zeichnet sich damit eher eine Kumulation ungleicher Verfügungsmöglichkeiten über Zeit im Lebensverlauf ab als deren Auflösung. Die Ziele einer stärker demografie- und lebenslauforientierten Arbeitszeitgestaltung und der Erhöhung der Arbeitsmarkt- und Beschäftigungsfähigkeit von benachteiligten Gruppen, wie weiblichen oder gering qualifizierten Beschäftigten, werden derzeit mit dem Langzeitkonto nicht ausreichend erreicht.

Welche Schlussfolgerungen lassen sich aus diesen Ergebnissen für die Arbeitszeitpolitik und zukünftige Forschung ziehen?

Die skizzierten Probleme unterstreichen zunächst, dass weiterhin ein großer Handlungsbedarf hinsichtlich der Einbettung und Ausgestaltung von Langzeitkonten besteht. Der Transaktionskostentheorie zufolge stellen institutionelle Arrangements eine wichtige Lösung für die skizzierten Probleme dar (Williamson 1985; Ebers / Gotsch 2006). Je mehr die mit der Nutzung von langfristig ausgelegten Arbeitszeitinstrumenten verbundenen Unsicherheiten und Risiken durch formelle und informelle Regelungen minimiert werden können, umso höher ist folglich die Chance, dass sie auch von bisher unterrepräsentierten Beschäftigtengruppen genutzt werden. Solche Regelungen betreffen nicht nur den Insolvenzschutz oder die Portabilität von Zeitguthaben, die in der Praxis nach wie vor ein Problem darstellen (vgl. Böker 2007; Hoff 2007). Erforderlich sind auch institutionelle Arrangements, die verbindliche Nutzungsansprüche definieren, eine langfristige Beschäftigungsperspektive stärken, Vertrauen zwischen den Betriebsparteien begründen und den Umgang mit Interessenkonflikten regeln. Tarifliche Regelungen können hier einen wichtigen Beitrag leisten, indem sie beispielsweise die Verwaltung und den Zugriff auf das Langzeitkonto auf überbetrieblicher Ebene regeln und damit eine stärkere Unabhängigkeit vom einzelnen Arbeitgeber gewährleisten. Die jüngsten Tarifverträge in der Chemie- und Metallindustrie lassen sich als wichtige Schritte in diese Richtung interpretieren. Darüber hinaus sind gezielte unterstützende Maßnahmen für niedrige Einkommensgruppen, Beschäftigte mit Betreuungs- oder Pflegeaufgaben und Beschäftigte in unsicheren Beschäftigtenverhältnissen notwendig, um auch diesen Gruppen den Aufbau ausreichender 
Zeitguthaben zu ermöglichen. Dass bei diesen Gruppen durchaus Interesse und Bedarf besteht, ein Langzeitkonto für Weiterbildung oder die bessere Vereinbarkeit von Beruf und Familie zu nutzen, hat die hier vorgestellte Untersuchung belegt.

Angesichts der skizzierten, zum Teil sehr massiven Einschränkungen in den zeitlichen Verfügungsmöglichkeiten bestimmter Beschäftigtengruppen stellt sich allerdings die grundsätzlichere Frage, ob das Langzeitkonto hier nicht deutlich überfordert wird. Um eine bessere Vereinbarkeit unterschiedlicher Lebensbereiche im Lebensverlauf zu ermöglichen ist die ganze Bandbreite bestehender Handlungsansätze und Instrumente der Arbeitszeitgestaltung gefragt, von Teilzeitoptionen über Sabbaticalmodelle bis hin zur täglichen flexiblen Arbeitszeitgestaltung. In der betrieblichen Praxis finden sich hierfür erfolgreiche Beispiele (Wotschack et al. 2011 a), die im Rahmen eines neuen arbeitszeitpolitischen Leitbildes weiter zu entwickeln und zu fördern wären (Wotschack et al. 2011 b). Individuelle Arbeitszeitoptionen für Phasen reduzierter Arbeitszeiten oder Freistellungen im Lebensverlauf entfalten dabei nur dann eine umfassende Wirkung, wenn die Norm der Arbeitszeitdauer und Leistungsanforderungen überhaupt Gestaltungsspielräume zulässt (Garhammer 2003; Wagner 2009). Dafür sind neben der gesetzlichen, tariflichen und betrieblichen Arbeitszeitgestaltung auch eine entsprechende Entgelt- und Leistungspolitik sowie eine ausreichende Personalausstattung der Unternehmen erforderlich. Individuelle Kontenmodelle (wie etwa Langzeitkonten), die auf dem Ansparen von Mehrarbeit und Entgeltbestandteilen beruhen, können nur dann zu einer umfassenden zeitlichen Entlastung der Beschäftigten beitragen, wenn die tariflichen oder vertraglichen Arbeitszeit- und Entgeltbedingungen es möglich machen, ausreichende Zeitguthaben anzusparen, ohne dadurch die alltägliche Balance von Erwerbsarbeit, Partnerschaft, Familie, Freizeit und Erholung langfristig zu gefährden (Hoff / Wotschack 2009).

Schließlich verweist die hier vorgestellte Untersuchung auf einen großen Bedarf nach Individualdaten zu Arbeitszeitmustern und Arbeitszeitpräferenzen. Die hier vorgestellten Befunde beruhen auf Beschäftigtendaten aus zwei Dienstleistungsgetrieben. Sie machen es zwar möglich, Unterschiede der Kontennutzung in Abhängigkeit von Merkmalen der beruflichen Stellung und des Geschlechts zu untersuchen, sind jedoch in ihrer Verallgemeinerbarkeit und Aussagekraft hinsichtlich branchen-, betriebs- oder haushaltsspezifischer Einflussfaktoren klar begrenzt. Repräsentative Individualdaten, die es ermöglichen würden, die Verbreitung, Nutzung und Wirkung unterschiedlicher Arbeitszeitinstrumente zu analysieren und auch im Längsschnitt zu verfolgen, liegen derzeit leider nicht vor. Hier zeigt sich ein großer Bedarf nach einer systematischen Arbeitszeitberichterstattung (vgl. auch Zapf 2012).

\section{Literatur}

Bauer, F. / H. Groß / G. Schilling (1994): Arbeitszeit '93, hrsg. vom Ministerium für Arbeit, Gesundheit und Soziales des Landes Nordrhein-Westfalen, Düsseldorf.

Bauer, F. / H. Groß / G. Schilling (1996): Arbeitszeit '95, hrsg. vom Ministerium für Arbeit, Gesundheit und Soziales des Landes Nordrhein-Westfalen, Düsseldorf.

Bauer, F. / H. Groß / K. Lehmann / E. Munz (2004): Arbeitszeit 2003. Arbeitszeitgestaltung, Arbeitsorganisation und Tätigkeitsprofile, Köln.

Benthaus-Apel, F. (1995): Zwischen Zeitbindung und Zeitautonomie, Wiesbaden.

Bolder, A. (2002): Arbeit, Qualifikation und Kompetenzen, in: R. Tippelt (Hrsg.), Handbuch Bildungsforschung, Opladen, S. 651-674.

Böker, K-H. (2007): Flexible Arbeitszeit - Langzeitkonten, Frankfurt / Main.

Bundesmann-Jansen, J. / H. Groß / E. Munz (2000): Arbeitszeit '99, hrsg. vom Ministerium für Arbeit des Landes Nordrhein-Westfalen, Köln. 
De Ruijter, E. / T. van der Lippe (2007): Household outsourcing: a transaction cost approach, in: T. van der Lippe / P. Peters (Hrsg.), Competing Claims in Work and Family Life, Cheltenham - Northampton, S. 195-211.

Durkheim, E. (1981): Die elementaren Formen des religiösen Lebens, Frankfurt / Main.

Ebers, M. / W. Gotsch (2006): Institutionenökonomische Theorien der Organisation, in: A. Kieser / M. Ebers (Hrsg.), Organisationstheorien, Stuttgart, S. 247-306.

Ehling, M. / N. Schwarz (1996): Zeit im Blickfeld, in: K. Blanke / M. Ehling / N. Schwarz (Hrsg.), Zeit im Blickfeld, Schriftenreihe des BMFSFJ, Bd 121, Stuttgart - Berlin - Köln.

Expertenkommission Finanzierung Lebenslangen Lernens (Hrsg.) (2004): Finanzierung Lebenslangen Lernens, hrsg. vom BMBF, Bielefeld.

Fischer, G. / J. Wahse / V. Dahms / M. Frei / A. Riedmann / F. Janik (2007): Standortbedingungen und Beschäftigung in den Regionen West- und Ostdeutschlands, IAB-Forschungsbericht 5 / 2007, Nürnberg.

Flüter-Hoffmann, C. (2010): Der Weg aus der Demografie-Falle, in: G. Naegele (Hrsg.), Soziale Lebenslaufpolitik, Wiesbaden, S. 411-428.

Garhammer, M. (1994): Balanceakt Zeit, Berlin.

Garhammer, M. (1999): Wie Europäer ihre Zeit nutzen, Berlin.

Garhammer, M. (2003): Neue Konzepte für lebensphasenspezifische Arbeitszeiten, in: H. Seifert (Hrsg.), Flexible Zeiten in der Arbeitswelt, Frankfurt / Main - New York.

Garhammer, M. (2007): Time pressure and quality of life, in: T. van der Lippe / P. Peters (Hrsg.), Competing Claims in Work and Family Life, Cheltenham - Northampton, S. 21-40.

Geißler, R. (Hrsg.)(1994): Soziale Schichtung und Lebenschancen in Deutschland, Stuttgart.

Gillen, J. / U. Elsholz / R. Meyer (2010): Soziale Ungleichheit in der beruflichen und betrieblichen Weiterbildung, Hans-Böckler-Stiftung, Arbeitspapier 191, Düsseldorf.

Groß, H. (1998): Überstundenabbau: Möglichkeiten von Schaffung und Sicherung von Beschäftigung, in: Loccumer Initiative (Hrsg.), Weniger Arbeit - weniger Demokratie? Kritische Interventionen, Bd 2, Hannover.

Groß, H. / M. Schwarz (2007): Betriebs- und Arbeitszeiten 2005, Sozialforschungsstelle Dortmund, Beiträge aus der Forschung, Band 153, Dortmund.

Groß, H. / M. Schwarz (2009): Arbeitszeit, Altersstrukturen und Corporate Social Responsibility, Wiesbaden.

Hildebrandt, E. (2007): Vorteile und Potenziale von Langzeitkonten, in: WSI-Mitteilungen 60, S. 620-622.

Hildebrandt, E. / P. Wotschack (2006): Langzeitkonten und Lebenslaufpolitik, in: WSI-Mitteilungen 59, S. 592-600.

Hildebrandt, E. / P. Wotschack / A. Kirschbaum (2009): Zeit auf der hohen Kante, Reihe Forschung aus der Hans-Böckler-Stiftung Bd 100, Berlin.

Hinrichs, K. (1982): Motive und Interessen im Arbeitszeitkonflikt. Frankfurt / Main - New York.

Hinrichs, K. (1988): Aufstieg und Niedergang von Normalarbeitsstandards, Frankfurt / Main - New York.

Hochschild, A. / A. Machung (1989): The Second Shift, New York.

Hoff, A. (2007): Warum auf Langzeit- und Lebensarbeitszeitkonten verzichtet werden sollte, in: WSIMitteilungen 60, S. 622-623.

Hoff, A. / P. Wotschack (2009): Kürzer Arbeiten, in: Personal 61, S. 6-8.

Institut der deutschen Wirtschaft (IW)(2006): Projektergebnisbericht: Lebenszyklusorientierte Personalpolitik, Studie des Instituts der deutschen Wirtschaft im Auftrag der DekaBank, Köln.

Jurczyk, K. (2010): Entgrenzte Arbeit - entgrenzte Familie, in: H. Groß / H. Seifert (Hrsg.), Zeitkonflikte, Berlin, S. 239-261. 
Kienbaum (2003): Zeitmanagement und Worklife Balance internationaler Top-Manager, Kienbaum Management Consultants, Berlin.

Kienbaum (2008): Kienbaum-Studie 2008. Personalentwicklung, Zürich.

Klenner, C. (2004): Erwartungen an einen familienfreundlichen Betrieb, in: Gewerkschaftliche Monatshefte 55, S. 452-458.

Klenner, C. / S. Pfahl (2009): Jenseits von Zeitnot und Karriereverzicht. Wege aus dem Arbeitszeitdilemma, in: M. Heitkötter / K. Jurczyk / A. Lange / U. Meier-Gräwe (Hrsg.), Zeit für Beziehungen?, Leverkusen.

Moen, P. (Hrsg.)(2003): It's About Time. Couples and Careers, Ithaca - London.

Müller-Wichmann, C. (1984): Zeitnot, Weinheim.

Munz, E. (2006): Mehr Balance durch selbst gesteuerte Arbeitszeiten?, in: WSI-Mitteilungen 59, S. 478-484.

Rosenbladt, B. von (1969): Tagesabläufe und Tätigkeitssysteme, in: Soziale Welt 20, S. 49-79.

Schimank, U. (2000): Handeln und Strukturen, Weinheim - München.

Schroeter, K.R. / Th. Rosenthal (2005): Soziologie der Pflege, Weinheim - München.

Schupp, J. / T. Gramlich / B. Isengard / R. Pischner / G.G. Wagner / B. von Rosenbladt (2003): Repräsentative Analyse der Lebenslagen einkommensstarker Haushalte, Studie des DIW, Berlin.

Schwarz, N. (1996): Zeit für unbezahlte Arbeit, in: K. Blanke / M. Ehling / N. Schwarz (Hrsg.), Zeit im Blickfeld, Schriftenreihe des BMFSFJ, Band 121, Stuttgart - Berlin - Köln, S. 70-91.

Seifert, H. (2003): Zeitenwende. Was bringen längere Arbeitszeiten für die Beschäftigung? In: WSI-Mitteilungen 56, S. 644-650.

Seifert, H. (2007): Arbeitszeit. Entwicklungen und Konflikte, in: APuZ 42, S. 17-24.

Seifert, H. (2010 a): Arbeitszeit- und Lernzeitkonten, in: G. Naegele (Hrsg.), Soziale Lebenslaufpolitik. Wiesbaden, S. 498-513.

Seifert, H. (2010 b): Beschäftigungsichernde Arbeitszeitverkürzungen, in: H. Groß / H. Seifert (Hrsg.), Zeitkonflikte, Berlin, S. 37-51.

Solga, H. (2005): Ohne Abschluss in die Bildungsgesellschaft, Opladen.

Solga, H. / J. Powel / P.A. Berger (Hrsg.)(2009): Soziale Ungleichheit, Frankfurt / Main - New York.

Sorokin, P.A. / R.K. Merton (1937): Social Time, in: American Journal of Sociology 42, S. 615-629.

Statistisches Bundesamt (1995): Die Zeitverwendung der Bevölkerung, Tabellenbände I-IV, Wiesbaden.

Statistisches Bundesamt (2003): Wo bleibt die Zeit?, Wiesbaden.

Van der Lippe, T. / P. Peters (2007): Competing Claims in Work and Family Life, Cheltenham - Northampton.

Wagner, A. (2000): Arbeitszeitwirklichkeit von (hoch-)qualifizierten Angestellten, in: S. Pfahl (Hrsg.), Moderne Arbeitszeiten für qualifizierte Angestellte?, Edition der Hans-Böckler-Stiftung 26, Düsseldorf.

Wagner, H. (2009): Der Arbeit ein gesundes Maß geben, in: IG Metall Projekt Gute Arbeit (Hrsg.), Handbuch „Gute Arbeit“, Hamburg.

Wiesenthal, H. (1987): Strategie und Illusion, Frankfurt / Main - New York.

Williamson, O.E. (1985): The Economic Institutions of Capitalism, New York.

Wotschack, P. (2002): Zeitwohlstand als Problem sozialer Ungleichheit, in: J. Rinderspacher (Hrsg.), Zeitwohlstand, Berlin, S. 143-163.

Wotschack, P. / E. Hildebrandt / F. Scheier (2008): Langzeitkonten. Neue Chancen für die Gestaltung von Arbeitszeiten und Lebensläufen?, in: WSI-Mitteilungen 61, S. 619-626. 
Wotschack, P. / Hildebrandt, E. (2008): Working-Life Time Accounts in German Companies, in: P. Ester / R. Muffels / J. Schippers / T. Wilthagen (Hrsg.), Innovating European Labour Markets. Cheltenham Northampton, S. 215-241.

Wotschack, P. (2010): Lebenslauforientierte Arbeitszeitgestaltung in Zeiten der Krise, in: H. Groß / H. Seifert (Hrsg.), Zeitkonflikte, Berlin, S. 195-212.

Wotschack, P. / F. Scheier / P. Schulte-Braucks / H. Solga (2011 a): Zeit für Lebenslanges Lernen. Neue Ansätze der betrieblichen Arbeitszeit- und Qualifizierungspolitik, in: WSI-Mitteilungen 64, S. 541-547.

Wotschack, P. / F. Scheier / P. Schulte-Braucks / H. Solga (2011 b): Beruf und Bildung vereinbaren. Neue Arbeitszeitmodelle gegen den Fachkräftemangel, WZBrief Arbeit 11, Berlin.

Zapf, I. (2012): Flexibilität am Arbeitsmarkt durch Überstunden und Arbeitszeitkonten. IAB-Forschungsbericht 3/2012, Nürnberg.

Dr. Philip Wotschack

Wissenschaftszentrum Berlin für Sozialforschung (WZB) Abteilung Ausbildung und Arbeitsmarkt

Reichpietschufer 50

10785 Berlin

wotschack@wzb.eu 


\section{Anhang}

Tabelle 4: Verbreitung des Langzeitkontos im Verkehrsdienstleistungsbetrieb (,,Ja, ich nutze das Langzeitkonto. "); logistische Regression (Odds Ratios)

\begin{tabular}{lcc} 
& $\mathrm{I}$ & $\mathrm{II}$ \\
\hline Konstante & $.228^{* *}$ & $.380^{* *}$ \\
Beschäftigtengruppe & & \\
Frau im administrativen Bereich & $2.767^{* *}$ & $2.522^{* *}$ \\
Mann im administrativen Bereich & $2.839^{* *}$ & $2.625^{* *}$ \\
Frau im operativen Bereich & .919 & .956 \\
Mann im operativen Bereich & Referenz & Referenz \\
Unsichere Zukunft (Selbsteinschätzung) & & \\
Index aus vier Items (siehe Tabelle 2) & & $.964^{* *}$ \\
\hline $\mathrm{N}$ & 4108 & 4108 \\
Nagelkerkes' $\mathrm{R}^{2}$ & .074 & .079 \\
\hline
\end{tabular}

(*) signifikant auf 10\%-Niveau; * signifikant auf 5\%-Niveau; ** signifikant auf 1\%-Niveau

Tabelle 5: Verbreitung des Langzeitkontos im IT-Betrieb (mindestens einmal Guthaben zwischen 2001 und 2007); logistische Regression (Odds Ratios)

\begin{tabular}{|c|c|c|c|c|c|}
\hline & I & II & III & IV & V \\
\hline \multirow{2}{*}{\multicolumn{6}{|c|}{ Geschlecht }} \\
\hline & & & & & \\
\hline Frau & .954 & 1.001 & 1.366 & 1.919 & 2.827 \\
\hline Mann & Ref. & Ref. & Ref. & Ref. & Ref. \\
\hline \multicolumn{6}{|l|}{ Beschäftigungsverhältnis } \\
\hline Vollzeit & .590 & 613 & $.562(*)$ & .576 & 617 \\
\hline Teilzeit & Ref. & Ref. & Ref. & Ref. & Ref. \\
\hline \multicolumn{6}{|l|}{ Alter } \\
\hline 19-45 Jahre & $.272 * *$ & $.271^{* *}$ & $.269 * *$ & $.347 * *$ & $.338 * *$ \\
\hline 46-64 Jahre & Ref. & Ref. & Ref. & Ref. & Ref. \\
\hline \multicolumn{6}{|l|}{ Stundensatz } \\
\hline Unter 30,10 Euro & $.576^{*}$ & $.576^{*}$ & $.656(*)$ & $.561 * *$ & $.622(*)$ \\
\hline $30,10-60,00$ Euro & Ref. & Ref. & Ref. & Ref. & Ref. \\
\hline \multicolumn{6}{|l|}{ Arbeitszeitmodell } \\
\hline Arbeitszeiterfassung & $3.198 * *$ & $3.197^{* *}$ & $3.276^{* *}$ & $3.260^{* *}$ & $3.322 * *$ \\
\hline Vertrauensarbeitszeit & Ref. & Ref. & Ref. & Ref. & Ref. \\
\hline \multicolumn{6}{|l|}{ Interaktionseffekte } \\
\hline Frau * Vollzeit & & .948 & & & .864 \\
\hline Frau $*$ Stundensatz unter 30 Euro & & & .578 & & .635 \\
\hline Frau * Alter von 19-45 Jahren & & & & $.389(*)$ & $.413(*)$ \\
\hline $\mathrm{N}$ & 745 & 745 & 745 & 745 & 745 \\
\hline Nagelkerkes' $\mathrm{R}^{2}$ & 197 & 197 & .200 & .204 & .205 \\
\hline
\end{tabular}

(*) signifikant auf 10\%-Niveau; * signifikant auf 5\%-Niveau; ** signifikant auf 1\%-Niveau 